\title{
Aggravation of Dysphagia Aortica After Wearing the Abdominal Binder
}

\author{
Seon-Young Park, MD, Jeoung-Hyeon Lee, MD, Sung-Bum Cho, MD, Wan-Sik Lee, MD, Chang-Hwan Park, MD, Hyun-Soo \\ Kim, MD, Sung-Kyu Choi, MD, Jong-Sun Rew, MD and Young-Eun Joo, MD* \\ Department of Internal Medicine, Chonnam National University Medical School, Gwangju, Korea
}

Dysphagia aortica is a rare etiology of dysphagia resulting from extrinsic compression of esophagus by thoracic aortic aneurysm or tortuosity and elongation of thoracic aorta. The clinical findings resemble those of esophageal malignancy or esophageal motility disorders. Therefore, primary diagnosis of dysphagia aortica is very difficult. We, herein, report a case of dysphagia aortica aggravated by wearing the abdominal binder in a 70-year-old woman and review the literature pertaining to this condition. Dysphagia aortica should be considered in the differential diagnosis of dysphagia.

(J Neurogastroenterol Motil 2010;16:323-326)

\section{Key Words}

Deglutition disorder, Aortica, Manometry, Abdomen, Equipment

\section{Introduction}

The term, dysphagia aortica, has been used to describe difficulty in swallowing caused by external compression of esophagus from an ectatic, tortuous, atherosclerotic or aneurysmal aorta. This entity is a rare condition and usually associated with old age, women with short stature, hypertension and kyphosis. ${ }^{1}$ To date, only 1 case has been recorded in South Korea. ${ }^{2}$ We, herein, report an additional case of dysphagia aortica aggravated by wearing the abdominal binder in a 70-year-old woman and review the literature pertaining to this condition.

\section{Case Report}

A 70-year-old woman visited to our department with a 3-month history of progressive dysphagia. She complained of mild nausea but no vomiting, abdominal pain or weight loss. She had mild dysphagia for 5 years but the severity of dysphagia did not worsen with time. Her past medical history revealed that she had been diagnosed with essential hypertension and spondylopathy. She developed low back pain due to spondylopathy, 4 months ago and was wearing the abdominal binder for relief of pain. Physical examination was revealed unremarkable. Her hematological and biochemical investigations were within normal range. Upper gastrointestinal endoscopy showed a pulsatile extrinsic compression at about $40 \mathrm{~cm}$ from incisor (Fig. 1). The underlying esophageal mucosa appeared entirely normal. Endo-

Received: June 18, 2010 Revised: June 24, 2010 Accepted: June 28, 2010

(c) This is an Open Access article distributed under the terms of the Creative Commons Attribution Non-Commercial License (http://creativecommons. org/licenses/by-nc/3.0) which permits unrestricted non-commercial use, distribution, and reproduction in any medium, provided the original work is properly cited.

*Correspondence: Young-Eun Joo, MD

Department of Internal Medicine, Chonnam National University Medical School, 8 Hak-dong, Dong-gu, Gwangju 501-757, Korea

Financial support: None. Tel: +82-62-220-6296, Fax: +82-62-225-8578, E-mail: yejoo@chonnam.ac.kr

Conflicts of interest: None. 


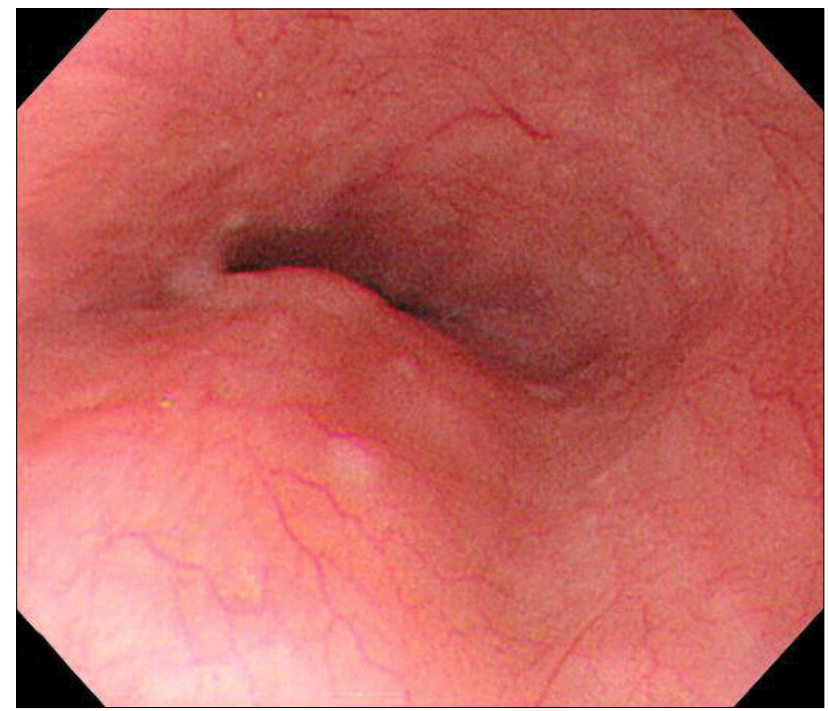

Figure 1. Upper gastrointestinal endoscopic finding. Endoscopy shows a pulsatile extrinsic compression at about $40 \mathrm{~cm}$ from the incisor. The underlying esophageal mucosa appears entirely normal. Endoscope has been introduced through the narrowing lumen with mild resistance.

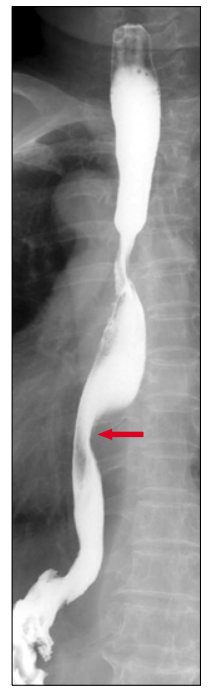

Figure 2. Upper gastrointestinal barium study. An upper gastrointestinal barium study shows the extrinsic indentation of distal esophagus (arrow) and mild passage disturbance.

scope was introduced through the narrowing lumen with mild resistance. An upper gastrointestinal barium study showed the extrinsic indentation of distal esophagus and mild passage disturbance (Fig. 2). The chest computed tomography showed the enlargement of left atrium and tortuous thoracic aorta with mild compression into adjacent esophagus (Fig. 3). Esophageal manometry revealed a localized high pressure barrier with super-

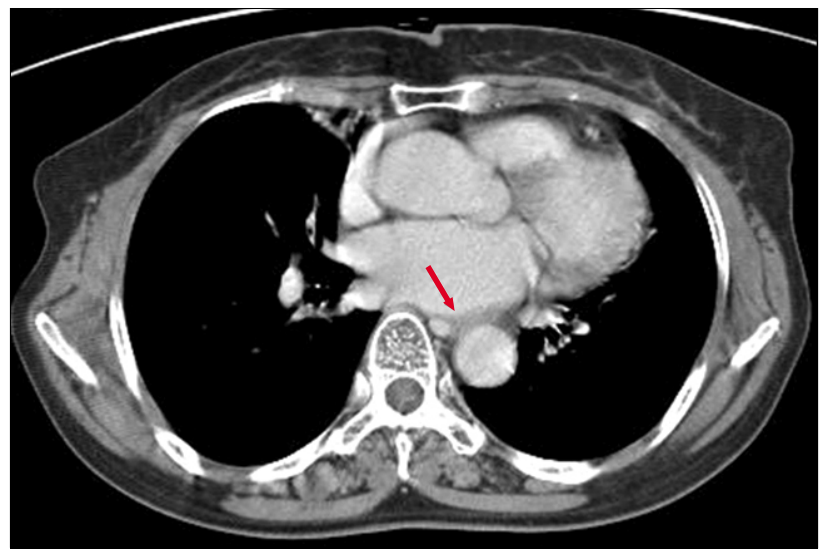

Figure 3. Chest computed tomography $(\mathrm{CT})$. The chest $\mathrm{CT}$ scan shows the enlargement of left atrium and tortuous thoracic aorta with mild compression into adjacent esophagus (arrow).

imposed vascular pulsation seen in only 1 transducer (Fig. 4A, arrow). The site of localized high pressure barrier was consistent with the site of luminal narrowing at endoscopic finding. And also, intermittent interruption of peristalsis (50\% nontransmitted contraction) and low amplitude of esophageal body were noted (Fig. 4A, arrowhead). However, manometry showed more improvement of peristaltic swallow activity and amplitude of esophageal body after taking off her abdominal binder (Fig. 4B). We instructed her to taking off the abdominal binder at meals for relieving dysphagia symptom. After modification of her diet and taking off the abdominal binder at meals, dysphagia was partially improved 2 months later.

\section{Discussion}

Dysphagia aortica is an uncommon type of dysphagia that is caused by extrinsic mechanical compression of esophagus due to an ectactic, tortuous, atherosclerotic or aneurysm aorta. Extrinsic compression causing esophageal dysphagia is also caused by numerous clinical entities, including cervical osteophytes, intrathoracic vascular anomalies (dysphagia lusoria), mediastinal tumors and an enlarged left atrium. ${ }^{3-7}$ In our case, the chest computed tomography showed the enlargement of left atrium and tortuous thoracic aorta with mild compression into adjacent esophagus.

Dysphagia aortica is classically seen in elderly women with short stature, hypertention and kyphosis. ${ }^{1}$ Our case was a 70 -year-old woman with short stature $(153 \mathrm{~cm})$ and hypertension.

The clinical symptoms of dysphagia aortica resemble those of 
B

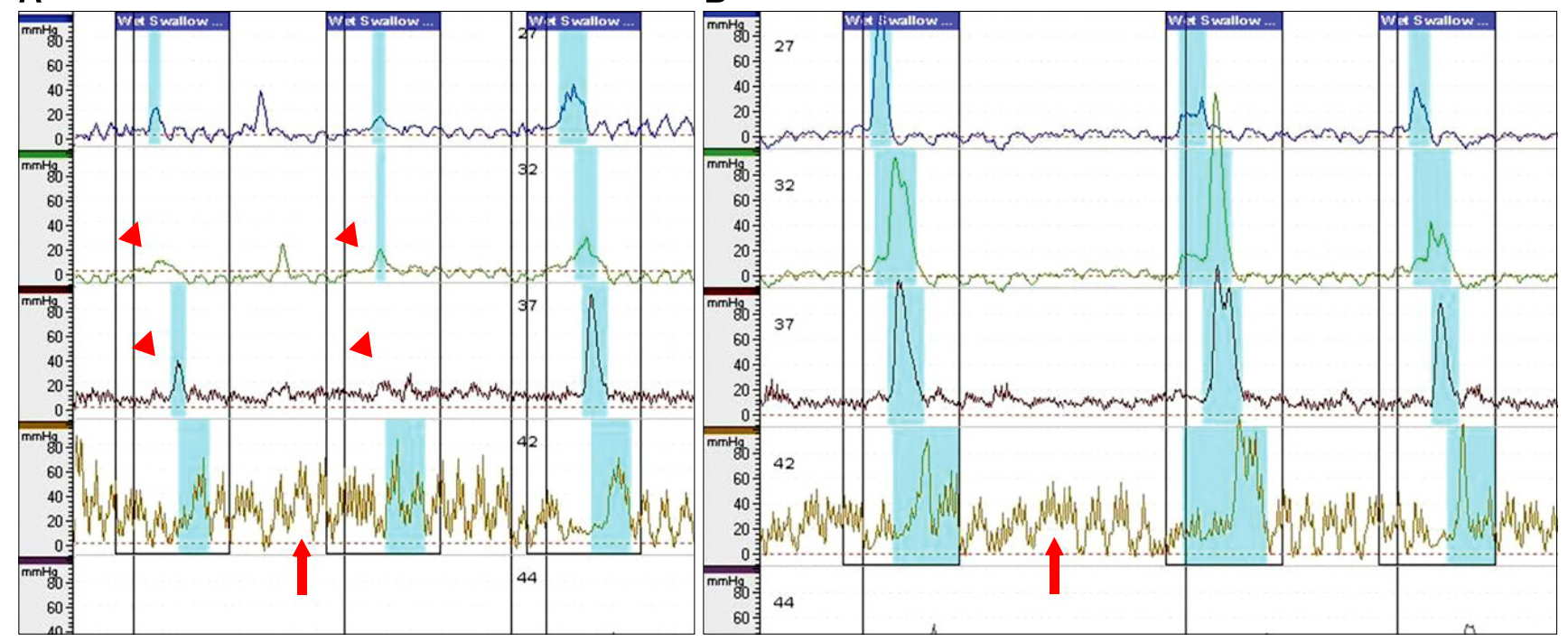

Figure 4. Esophageal manometric findings. (A) Esophageal manometry reveals a localized high pressure barrier (arrow) with superimposed oscillations synchronous with aortic pulsating typically seen in only one transducer. And also, intermittent interruption of peristalsis of (50\% nontransmitted contraction) and low amplitude of esophageal body are noted (arrowhead). (B) Manometry shows more improvement peristaltic swallow activity and low amplitude of esophageal body after taking off her abdominal binder.

esophageal malignancy or esophageal motility disorders. Also, symptoms of dysphagia aortica are usually mild and indolent. Therefore, primary diagnosis of dysphagia aortica is very difficult. Our case had mild dysphagia for 5 years but the severity of dysphagia did not worsen with time. However, we could diagnose the dysphagia aortica due to aggravation of dysphagia symptom after wearing the abdominal binder. Therefore, a high index of clinical suspicion is important in identifying patient with dysphagia aortica. ${ }^{8}$ And the dramatic difference in the prognosis and treatment between esophageal malignancy or esophageal motility disorders and dysphagia aortica stresses the importance of differential diagnosis of dysphagia.

There is no gold standard diagnostic procedure for dysphagia aortica. In previous reports, the diagnostic approach of dysphagia aortica includes standard chest radiography, chest computed tomography, barium swallow test and esophageal manometry. In our case, all diagnostic procedures were done. The findings of diagnostic procedures were similar with those of previous reports. ${ }^{1,9,10}$

Esophageal manometry of our case revealed a localized high pressure barrier with superimposed vascular pulsation, intermittent interruption of peristalsis and low amplitude of esophageal body. ${ }^{10}$ However, intermittent interruption of peristalsis and amplitude of esophageal body were improved after taking off her abdominal binder. It is possible that wearing the abdominal binder may promote intra-abdominal pressure. Extrinsic abdominal compression may lead to a simultaneous increase of pressure in the stomach, LES and esophagus (common cavity phenomenon). ${ }^{11}$ So, the increase of abdominal pressure may lead to retard peristaltic swallowing activity. And, also, the increased abdominal pressure may lead to subsequent relaxation of the lower esophageal sphincter, thus facilitating abnormal reflux. ${ }^{12}$ So, her abdominal binder may play a role in causing previously subclinical extrinsic compression of esophagus to become symptomatic.

The treatment of dysphagia aortica depends on the severity of symptoms. Most cases are mild in nature and can be treated conservatively. Many surgical procedures have been described for the management of severe cases. ${ }^{1}$ In our case, dysphagia symptom was improved by modification of diet and taking off the abdominal binder at meals.

In summary, first, dysphagia aortica, despite its rarity, should be considered in the differential diagnosis of dysphagia. Second, to wear the abdominal binder affects the esophageal motility by increasing the intra-abdominal pressure.

\section{References}

1. Wilkinson JM, Euinton HA, Smith LF, Bull MJ, Thorpe JA. Diagnostic dilemmas in dysphagia aortica. Eur J Cardiothorac Surg 
1997;11:222-227.

2. Kim JH, Jang SW, Kim DB, et al. A patient with dysphagia due to an aortic aneurysm. Korean Circ J 2009;39:258-260.

3. Taylor CW, Sinha A, Nightingale JM. Dysphagia and thoracoabdominal aneurysm. Postgrad Med J 2001;77:257-258.

4. Jougon JB, Lepront DJ, Dromer CE. Posterior dislocation of the sternoclavicular joint leading to mediastinal compression. Ann Thorac Surg 1996;61:711-713.

5. Ramakantan R, Shah P. Dysphagia due to mediastinal fibrosis in advanced pulmonary tuberculosis. AJR Am J Roentgenol 1990;154: 61-63.

6. Dantas RO, de Godoy RA, Meneghelli UG, de Oliveira RB, Troncon LE. Dysphagia lusoria and segmental aperistalsis in the upper third of the esophagus. J Clin Gastroenterol 1985;7:522-524.
7. Morgan AA, Mourant AJ. Left vocal cord paralysis and dysphagia in mitral valve disease. Br Heart J 1980;43:470-473.

8. Hilliard AA, Murali NS, Keller AS. Dysphagia aortica. Ann Intern Med 2005;142:230-231.

9. Birnholz JC, Ferrucci JT, Wyman SM. Roentgen features of dysphagia aortica. Radiology 1974;111:93-96.

10. Mittal RK, Siskind BN, Hongo M, Flye MW, McCallum RW. Dysphagia aortica. Clinical, radiological, and manometric findings. Dig Dis Sci 1986;31:379-384.

11. Butterfield DG, Struthers JE Jr, Showalter JP. A test of gastroesophageal sphincter competence. The common cavity test. Am J Dig Dis 1972;17:415-421.

12. Ott DJ. Motility disorders of the esophagus. Radiol Clin North Am 1994;32:1117-1134. 